\title{
Caractéristiques physico-chimiques de l'eau des puits dans la commune de Pobè (Bénin, Afrique de l'ouest)
}

\author{
Moïssou LAGNIKA ${ }^{1,2^{*}}$, Moudachirou IBIKOUNLE1, Jean-Pierre C. MONTCHO ${ }^{2}$, Valentin D. WOTTO³, \\ Nestor G. SAKITI 1 \\ 'Département de zoologie, Faculté des Sciences et Techniques, Université d'Abomey-Calavi, 01 BP : 526 Cotonou, \\ Bénin. \\ 2Laboratoire d'analyses des eaux, Service de l'Eau du Borgou, Direction Départementale de l'Énergie et de l'Eau, \\ BP : 197 Parakou, Bénin. \\ ${ }^{3}$ Laboratoire de Chimie Physique, Faculté des Sciences et Techniques, Université d'Abomey Calavi, 02 BP : 353 \\ Cotonou, Bénin. \\ *Auteur correspondant, E-mail : moissou@yahoo.fr
}

Original submitted in on $23^{\text {rd }}$ April 2014. Published online at www.m.elewa.org on $31^{\text {st }}$ July 2014. http://dx.doi.org/10.4314/jab.v79i1.13

\section{RÉSUMÉ}

Objectif : L'objectif de cette étude est d'évaluer la qualité de l'eau des puits à partir de ses caractéristiques physico-chimiques dans la commune de Pobè.

Méthodologie et résultats : L'étude entreprise entre juin 2012 et mars 2013 a permis de mesurer au niveau de quinze puits les paramètres physiques tels que le $\mathrm{pH}$, la conductivité électrique (CE) et la température (T) de l'eau ainsi que les paramètres chimiques suivants : bicarbonates $\left(\mathrm{HCO}_{3}^{-}\right)$, chlorures $(\mathrm{Cl}-)$, calcium $\left(\mathrm{Ca}^{2+}\right)$, magnésium $\left(\mathrm{Mg}^{2+}\right)$, titre hydrotimétrique ou dureté totale $(\mathrm{TH})$, ammonium $\left(\mathrm{NH}_{4}{ }^{+}\right)$, nitrates $\left(\mathrm{NO}_{3}{ }^{-}\right)$, nitrites $\left(\mathrm{NO}_{2}{ }^{-}\right)$, sulfates $\left(\mathrm{SO}_{4}{ }^{-}\right)$, phosphates $\left(\mathrm{PO}_{4}{ }^{3-}\right)$ et fluorures $\left(\mathrm{F}^{-}\right)$. Les valeurs moyennes annuelles sont comparées aux normes relatives à la qualité de l'eau de boisson. L'analyse statistique multivariée dont l'Analyse en Composantes Principales (ACP) et la Classification Hiérarchique Ascendante (CHA) a été également appliquée à l'ensemble des paramètres mesurés. L'eau des puits est légèrement acide avec un $\mathrm{pH}$ moyen de $5,83 \pm 0,6$. Elle est faiblement minéralisée et légèrement dure avec une conductivité moyenne de $236,62 \pm 135,54 \mu \mathrm{S} / \mathrm{cm}$ et une dureté totale moyenne de 107,8 $\pm 40,63 \mathrm{mg} / \mathrm{L}$. La pollution azotée est un indicateur majeur de la qualité de l'eau des puits étudiés. Cette pollution se traduit par des taux élevés en nitrates et en ammonium dans plus de $50 \%$ des puits. Une forte corrélation est signalée entre la conductivité électrique et les paramètres suivants : $\mathrm{pH}, \mathrm{NO}_{3}{ }^{-}, \mathrm{NO}_{2}^{-}, \mathrm{SO}_{4}^{2-}, \mathrm{TH}, \mathrm{Cl}, \mathrm{HCO}_{3}{ }^{-}$et $\mathrm{Ca}^{2+}$. La typologie est principalement gouvernée par cette conductivité qui permet de classifier les puits selon leur qualité physico-chimique.

Conclusion et applications : L'eau des puits est soumise à une pollution organique dans la commune de Pobè. Cette pollution d'origine anthropique serait liée à l'infiltration des eaux usées et des engrais chimiques. Les fortes teneurs en nitrates et en ammonium montrent que cette eau est chimiquement non appropriée à la consommation humaine et nécessite dans la majorité des puits un traitement préalable.

Mots clés : Physico-chimie, eau de puits, pollution, analyses multivariées, Pobè. 
Physicochemical characteristics of well water in Pobè municipality, Benin, West Africa

\section{ABSTRACT}

Objective: The aim of this study is to evaluate the quality of well water for its physical and chemical characteristics in the municipality of Pobè.

Methodology and Results: The study conducted from June 2012 to March 2013 measured in fifteen wells the level of physical parameters such as $\mathrm{pH}$, electrical conductivity and water temperature as well as the following chemical parameters: bicarbonate $\left(\mathrm{HCO}_{3}{ }^{-}\right)$, chloride $(\mathrm{Cl})$, calcium $\left(\mathrm{Ca}^{2+}\right)$, magnesium $\left(\mathrm{Mg}^{2+}\right)$, total hardness $(\mathrm{TH})$, ammonium $\left(\mathrm{NH}_{4}^{+}\right)$, nitrate $\left(\mathrm{NO}_{3}^{-}\right)$, nitrite $\left(\mathrm{NO}_{2}^{-}\right)$, sulphate $\left(\mathrm{SO}_{4}^{2-}\right)$, phosphate $\left(\mathrm{PO}_{4}^{3-}\right)$ and fluoride $\left(\mathrm{F}^{-}\right)$. The annual mean values are compared to standards for drinking water quality. Statistical multivariate analysis including Principal Component Analysis and hierarchical clustering was also applied to all measured parameters. Well water is slightly acidic with an average pH of $5.83 \pm 0.6$. It is weakly mineralized and slightly hard with an average of $236.62 \pm 135.54 \mu \mathrm{S} / \mathrm{cm}$ electrical conductivity and a total hardness of $107.8 \pm 40.63 \mathrm{mg} / \mathrm{L}$. Nitrogen pollution is a major indicator of well water quality. This pollution finds expression in high rates of nitrate and ammonium in more than $50 \%$ of the wells. It was observed that electrical conductivity show good positive correlation with $\mathrm{pH}, \mathrm{NO}_{3}^{-}, \mathrm{NO}_{2}^{-}, \mathrm{SO}_{4}^{2-}, \mathrm{TH}, \mathrm{Cl}^{-}, \mathrm{HCO}_{3}^{-}$and $\mathrm{Ca}^{2+}$.The typology is mainly governed by the conductivity for classifying the wells according to their physical and chemical quality.

Conclusion and applications : Well water is subjected to organic pollution in the municipality of Pobè. This anthropogenic pollution is linked to the infiltration of sewage and chemical fertilizers. The high levels of ammonium and nitrate indicate that the water is chemically unsuitable for human consumption and requires that water in most wells be pretreated.

Keywords : Physicochemical, well water, pollution, multivariate analysis, Pobè.

\section{INTRODUCTION}

L'eau constitue une ressource naturelle indispensable dans la vie des hommes, des animaux et des végétaux. L'avoir à disposition en quantité suffisante et en qualité contribue au maintien de la santé. Mais elle peut être aussi source de maladies du fait de sa pollution par des rejets industriels, des eaux usées, des déchets ménagers ou agricoles, des excrétas et divers déchets organiques (Scalon et al., 2005 ; El-Naqa et al., 2007; Eblin et al., 2014 ). Selon l'Organisation Mondiale de la Santé, environ 1,1 milliard de personnes n'ont pas accès à l'eau potable et 2,4 milliards n'ont pas accès à un système d'assainissement adéquat. Plus de 2 millions de personnes, surtout des enfants de moins de cinq ans des pays en développement où les mesures d'hygiène et d'assainissement sont insuffisantes, meurent chaque année des maladies diarrhéiques (OMS, 2011). Or, à l'échelle mondiale, près de $90 \%$ des maladies diarrhéiques sont imputables à la mauvaise qualité de l'eau de boisson et à un assainissement insuffisant des eaux usées. L'eau est devenue aujourd'hui un enjeu stratégique mondial dont la gestion doit impérativement s'intégrer dans une perspective politique de développement durable (Servais et al., 2009). Ainsi, l'approvisionnement en eau potable des populations en quantité suffisante en toutes saisons demeure l'un des soucis majeurs des différents gouvernements (Hawa, 2002). L'eau de boisson est un élément vital dont les sources d'approvisionnement sont très diversifiées. Au Bénin, près d'un tiers de ménages utilise l'eau courante comme eau de boisson. $22,6 \%$ des ménages s'approvisionnent en eau de puits non protégés et en eau des rivières $(13,2 \%)$ qui sont des sources d'eau non potable. Les ménages s'approvisionnent aussi en eau de fontaine et des pompes villageoises $(21,2 \%)$ et en eau de puits protégés dans $8,3 \%$ des cas (INSAE, 2003). Une eau non potable nécessite un traitement avant sa consommation. Le traitement de l'eau regroupe les principales opérations suivantes : la coagulation, la floculation, la sédimentation, la filtration et la 
désinfection (Fatombi et al., 2012). Le département du plateau et plus particulièrement la commune de Pobè fait partie des communes les moins desservies par la Société Nationale des Eaux du Bénin (SONEB). En effet, sur les cinq arrondissements que compte cette commune, un seul est en partie desservi par cette société. Les populations ont recours à l'eau des forages et surtout à celle des puits dans l'ensemble des arrondissements. Si l'eau provenant des forages

\section{MATÉRIEL ET MÉTHODES}

Zone d'étude : La commune de Pobè est limitée au Nord par la commune de Kétou, au sud et à l'Ouest par la commune d'Adja-Ouèrè et à l'est par le Nigéria (Fig.1). Elle a une superficie de $400 \mathrm{~km}^{2}$ représentant $0,46 \%$ de celle du Bénin. Elle est composée de quarante quatre villages et quartiers de ville qui sont répartis dans cinq arrondissements dont un urbain et quatre ruraux. Le climat est de type subéquatorial avec deux saisons pluvieuses et deux saisons sèches qui s'alternent. Les précipitations atteignent 1100 à 1200 $\mathrm{mm}$ par an. Le relief est constitué par une zone de plateaux de 50 à 200 mètres au dessus du niveau de la mer et d'une altitude moyenne de 100 mètres avec une dépression qui n'est rien d'autre que la continuité de la dépression médiane qui traverse tout le Bénin de l'Ouest à l'Est. Elle découpe la commune en deux zones orographiques : une zone de dépression et une zone de plateau. La zone de dépression comporte une grande partie de l'arrondissement d'Issaba jusqu'à la latitude d'Onigbolo et les arrondissements d'Ahoyéyé, d'Igana et de Towé. Celle de plateaux regroupe tout le reste de la commune. Sur celle-ci, on rencontre plusieurs plateaux dont le plateau de Pobè ayant une altitude moyenne de 100 mètres. Dans la commune de Pobè, on distingue deux types d'unité pédologique : un sol hydromorphe et un sol ferralitique. Le sol hydromorphe très fertile où se pratiquent les principales cultures agricoles est situé dans la dépression d'Issaba. Cette zone riche en calcaire a permis est en général de bonne qualité, celle provenant des puits reste d'une qualité douteuse. En effet, les études réalisées sur la qualité de l'eau des puits dans d'autres régions du sud-Bénin ont montré qu'elle est impropre à la consommation humaine (Comlanvi,1994; Dégbey et al., 2008; Makoutodé et al.,1999). La présente étude a été entreprise afin d'évaluer la qualité physicochimique de l'eau provenant des puits dans la commune de Pobè.

l'implantation de l'usine cimentière gérée par la société Lafarge à Onigbolo. Le sol ferralitique situé sur le plateau Pobè-Sakété est composé de sable, de grès et d'argile. C'est un sol rouge occupant le quart (1/4) de la superficie de la commune. Le réseau hydrographique est quasi nul mais quelques ruisseaux sont presque permanents tels que la réserve botanique de l'ex. IRHO, l'Itchèko, Itché, Iwin, Ebé, Idi et Ikpori. Aussi des retenues d'eau importantes sont relevées pendant la saison des pluies (Bani, 2006).

Choix des stations : Quinze stations sélectionnées sont constituées de puits répartis sur l'ensemble des cinq arrondissements (Fig.1). Cinq d'entre eux sont choisis dans l'arrondissement urbanisé et les autres répartis dans les arrondissements ruraux. Le tableau 1 présente les différentes caractéristiques des stations étudiées. Parmi les quinze puits, les puits $\mathrm{Pb} 2, \mathrm{~Pb} 3$, $\mathrm{Pb} 4, \mathrm{~Pb} 5, \mathrm{~Pb} 9, \mathrm{~Pb} 12, \mathrm{~Pb} 13$ et $\mathrm{Pb} 14$ sont équipés chacun d'une pompe électrique et l'eau est vendue aux populations grâce à ces postes d'eau autonomes installés par des particuliers. Un poste d'eau autonome par définition est un ensemble d'équipements électrique, électromécanique et hydraulique destiné à pomper, stocker et distribuer de l'eau. L'autonomie traduit l'existence d'un temps pendant lequel l'approvisionnement en eau se fait sans nouvel apport d'énergie et sans intervention extérieure (Ingabiré Zannou, 2006). 


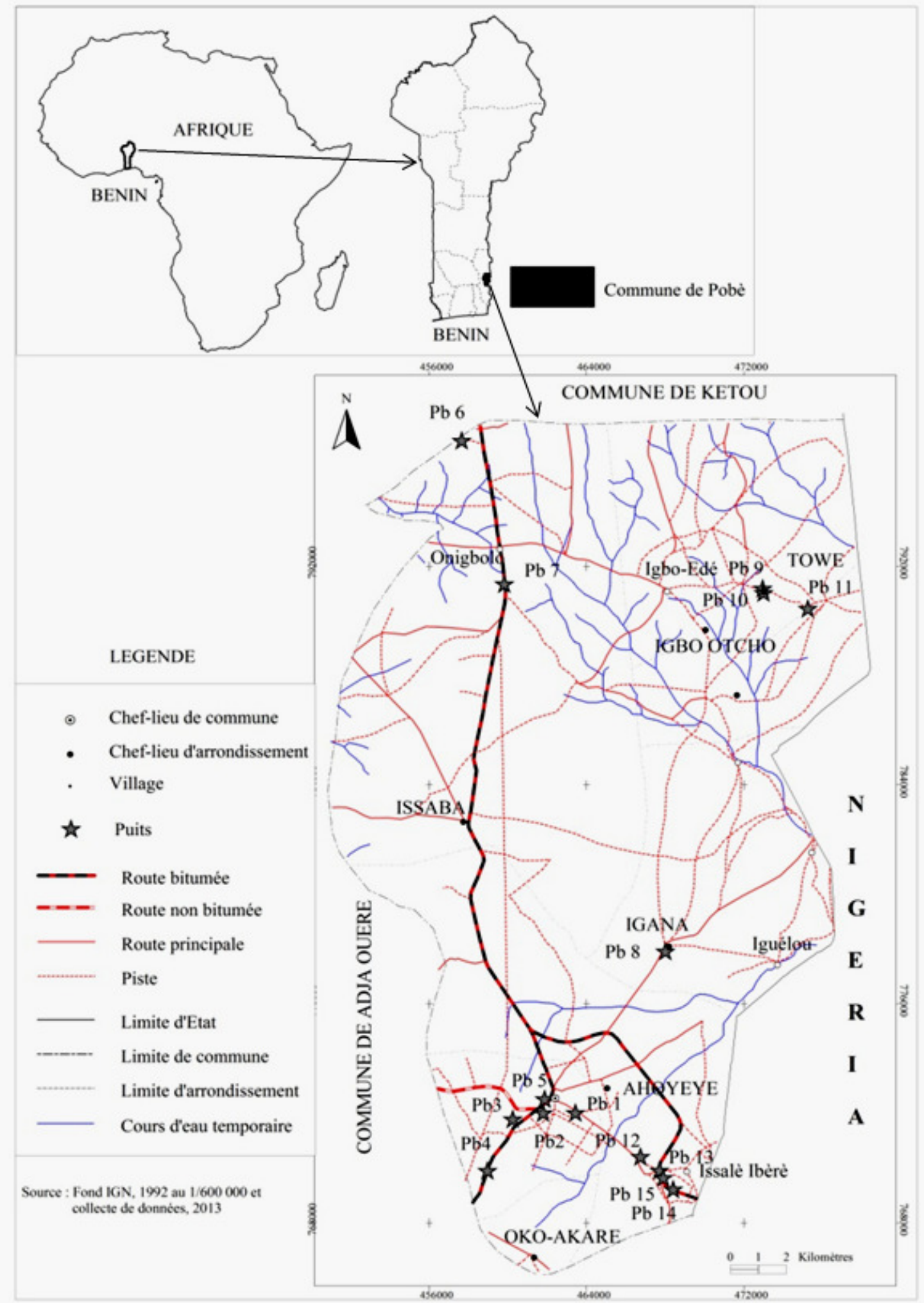

Figure 1 : Carte de la zone d'étude avec indication des stations 


\section{Lagnika et al. J. Appl. Biosci. 2014. Caractéristiques physico-chimiques de l'eau des puits dans la}

commune de Pobè, Benin

Tableau 1 : Description des caractéristiques et environnement des stations étudiées

\begin{tabular}{l|l|l|l|l|l}
\hline Stations & $\begin{array}{l}\text { Profondeur } \\
\text { totale }(\mathrm{m})\end{array}$ & $\begin{array}{l}\text { Margelle } \\
(\mathrm{m})\end{array}$ & $\begin{array}{l}\text { Etat de la paroi du } \\
\text { puits }\end{array}$ & $\begin{array}{l}\text { Couverture du } \\
\text { toit }\end{array}$ & $\begin{array}{l}\text { Source probable de } \\
\text { pollution de l'eau }\end{array}$ \\
\hline Pb1 & 17.30 & 0.80 & Non cimenté & Sans couverture & Infiltration des eaux \\
\hline Pb2 & 36.08 & 0.80 & Cimenté & Dalle & Proximité des latrines \\
\hline Pb3 & 40.80 & 0.80 & Cimenté & Dalle & Non identifiée \\
\hline Pb4 & 50.50 & 0.80 & Cimenté & Dalle & Non identifiée \\
\hline Pb5 & 16.20 & 0.50 & Cimenté & Dalle & Infiltration des eaux \\
\hline Pb6 & 19.60 & 0.40 & Cimenté & Planches & Agriculture \\
\hline Pb7 & 6.10 & 0.28 & Non cimenté & Tôle & Agriculture \\
\hline Pb8 & 26.27 & 0.90 & Cimenté & Sans couverture & Agriculture \\
\hline Pb9 & 19.25 & 0.95 & Cimenté & Bois & Agriculture \\
\hline Pb10 & 17.60 & 0.70 & Cimenté & Sans couverture & Infiltration des eaux \\
\hline Pb11 & 13.90 & 0.80 & Non cimenté & Tôle & Infiltration des eaux \\
\hline Pb12 & 14.90 & 0.25 & Cimenté & Dalle & Non identifiée \\
\hline Pb13 & 34.52 & 0.20 & Cimenté & Dalle & Non identifiée \\
\hline Pb14 & 36.00 & 0.85 & Cimenté & Dalle & Proximité des toilettes \\
\hline Pb15 & 31.00 & 0.60 & Cimenté & Sans couverture & Non identifiée \\
\hline
\end{tabular}

Échantillonnage et analyse de l'eau : L'échantillonnage a été réalisé entre juin 2012 et mars 2013. Quatre prélèvements ont été réalisés par puits et par saison. Les prélèvements ont été effectués au niveau de chaque puits à l'aide d'un petit seau lesté puis conservés pendant 72 heures au frais dans des plastiques d'eau minérale de $1,5 \mathrm{~L}$ pour les analyses. Toutefois, le $\mathrm{pH}$, la conductivité et la température ont été mesurés sur le terrain grâce à un multimètre portatif (HANNA, HI 991300). Les bicarbonates, les chlorures, le calcium, le magnésium et la dureté totale ont été mesurés par titrimétrie tandis que l'ammonium, les nitrates, nitrites, sulfates, phosphates et fluorures ont

\section{RÉSULTATS ET DISCUSSION}

Caractéristiques physico-chimiques de l'eau : Les résultats des analyses physico-chimiques de l'eau des puits étudiés sont consignés dans le tableau 2. Le tableau 3 regroupe les normes de qualité de l'eau de boisson, les valeurs moyennes et extrêmes des différents paramètres physico-chimiques mesurés de l'eau des puits. La température de l'eau des puits de été déterminés par un spectrophotomètre $(\mathrm{HACH}, \mathrm{DR}$ 2400) conformément aux méthodes préconisées par Rodier (2009).

Analyses statistiques : L'Analyse en Composantes Principales a été réalisée sur les valeurs moyennes annuelles des paramètres physico-chimiques caractérisant les quinze puits (Tableau 2). Cette analyse a permis de faire ressortir les corrélations existantes entre les différents paramètres de l'eau. Afin d'obtenir une meilleure affectation des individus à leur classe, cette analyse a été complétée par une Classification Hiérarchique Ascendante telle que préconisée par Belkhiri et al. (2010).

Pobè est en moyenne de $28,15 \pm 0,32{ }^{\circ} \mathrm{C}$. Elle est comprise entre $27,6^{\circ} \mathrm{C}$ et $28,7^{\circ} \mathrm{C}$. La valeur obtenue est similaire à celle obtenue par Nwala et al. (2007) et par Dégbey et al. (2008). La conductivité électrique a donné des valeurs comprises entre 74,8 et 538,2 $\mu \mathrm{S} / \mathrm{cm}$ avec une moyenne de $236,62 \pm 135,54 \mu \mathrm{S} / \mathrm{cm}$ pour l'ensemble des puits étudiés. 
Tableau 2 : Valeurs moyennes annuelles des paramètres physico-chimiques de l'eau des quinze puits

\begin{tabular}{|c|c|c|c|c|c|c|c|c|c|c|c|c|c|c|}
\hline $\begin{array}{l}\text { Variables } \\
\text { Puits }\end{array}$ & $\begin{array}{l}\mathrm{T} \\
{ }^{\circ} \mathrm{C}\end{array}$ & $\begin{array}{l}\text { C E } \\
\mu S / c m\end{array}$ & $\mathrm{pH}$ & $\begin{array}{l}\mathrm{NO}_{3}^{-} \\
\mathrm{mg} / \mathrm{L}\end{array}$ & $\begin{array}{l}\mathrm{NO}_{2}^{-} \\
\mathrm{mg} / \mathrm{L}\end{array}$ & $\begin{array}{l}\mathrm{NH}_{4}^{+} \\
\mathrm{mg} / \mathrm{L}\end{array}$ & $\begin{array}{l}\mathrm{SO}_{4}^{2-} \\
\mathrm{mg} / \mathrm{L}\end{array}$ & $\begin{array}{l}\mathrm{PO}_{4}^{3-} \\
\mathrm{mg} / \mathrm{L}\end{array}$ & $\mathrm{Fg} \cdot \mathrm{L}$ & $\begin{array}{l}\mathrm{Ca}^{2+} \\
\mathrm{mg} / \mathrm{L}\end{array}$ & $\begin{array}{l}\mathrm{Mg}^{2+} \\
\mathrm{mg} / \mathrm{L}\end{array}$ & $\begin{array}{l}\mathrm{TH} \\
\mathrm{mg} / \mathrm{L}\end{array}$ & $\begin{array}{l}\mathrm{Cl}^{-} \\
\mathrm{mg} / \mathrm{L}\end{array}$ & $\begin{array}{l}\mathrm{HCO}_{3}{ }^{-} \\
\mathrm{mg} / \mathrm{L}\end{array}$ \\
\hline $\mathrm{Pb} 1$ & 27,6 & 538,2 & 6,19 & 91,7 & 0,598 & 0,388 & 26,0 & 2,84 & 0,05 & 41,76 & 12,90 & 149,0 & 67,45 & 35,07 \\
\hline Pb2 & 28,1 & 288,5 & 5,29 & 107,0 & 0,023 & 0,009 & 8,5 & 1,46 & 0,01 & 22,34 & 18,77 & 98,5 & 50,14 & 13,72 \\
\hline Pb3 & 28,4 & 90,7 & 5,46 & 11,9 & 0,021 & 0,032 & 1,3 & 0,46 & 0,09 & 18,71 & 11,88 & 83,5 & 20,44 & 27,45 \\
\hline Pb4 & 28,4 & 153,1 & 5,17 & 14,9 & 0,012 & 0,009 & 1,0 & 0,80 & 0,03 & 11,35 & 9,80 & 80,0 & 36,38 & 17,53 \\
\hline Pb5 & 28,1 & 192,0 & 5,27 & 74,0 & 0,017 & 0,406 & 6,7 & 0,58 & 0,20 & 19,00 & 12,51 & 90,0 & 29,28 & 25,92 \\
\hline $\mathrm{Pb} 6$ & 28,2 & 310,0 & 6,16 & 99,8 & 0,019 & 0,088 & 3,8 & 0,36 & 0,11 & 18,43 & 13,02 & 103,0 & 30,17 & 29,73 \\
\hline Pb7 & 28,5 & 274,2 & 6,26 & 4,3 & 0,086 & 0,053 & 17,5 & 0,22 & 0,09 & 23,61 & 18,96 & 114,0 & 33,72 & 45,75 \\
\hline Pb8 & 27,7 & 287,7 & 6,87 & 6,8 & 0,011 & 1,087 & 17,0 & 0,65 & 0,52 & 41,46 & 20,39 & 150,0 & 31,95 & 58,71 \\
\hline $\mathrm{Pb} 9$ & 28,7 & 494,7 & 6,88 & 127,0 & 0,139 & 0,048 & 4,7 & 0,62 & 0,36 & 74,29 & 11,77 & 232,0 & 34,61 & 83,87 \\
\hline $\mathrm{Pb} 10$ & 28,7 & 320,5 & 6,66 & 62,1 & 0,038 & 0,126 & 8,3 & 0,94 & 0,09 & 59,31 & 5,81 & 121,0 & 25,74 & 59,47 \\
\hline $\mathrm{Pb} 11$ & 28,2 & 239,7 & 6,10 & 13,3 & 0,019 & 0,519 & 1,5 & 0,77 & 0,18 & 42,01 & 6,17 & 104,0 & 22,19 & 48,80 \\
\hline $\mathrm{Pb} 12$ & 28,1 & 112,5 & 5,42 & 9,0 & 0,013 & 0,023 & 0,7 & 0,47 & 0,13 & 9,89 & 10,17 & 54,0 & 23,07 & 32,02 \\
\hline $\mathrm{Pb} 13$ & 28,1 & 74,8 & 5,18 & 11,7 & 0,031 & 0,074 & 1,0 & 0,31 & 0,03 & 15,20 & 14,34 & 82,0 & 31,95 & 28,97 \\
\hline $\mathrm{Pb} 14$ & 27,7 & 76,0 & 5,32 & 16,9 & 0,029 & 0,026 & 0,5 & 0,35 & 0,15 & 16,55 & 15,02 & 70,5 & 17,75 & 31,26 \\
\hline $\mathrm{Pb} 15$ & 27,8 & 96,7 & 5,29 & 29,1 & 0,019 & 0,019 & 0,8 & 0,43 & 0,09 & 10,15 & 22,92 & 85,5 & 21,74 & 29,73 \\
\hline
\end{tabular}




\section{Lagnika et al. J. Appl. Biosci. 2014. Caractéristiques physico-chimiques de l'eau des puits dans la}

commune de Pobè, Benin

La faible minéralisation de l'eau des puits obtenue est contraire aux résultats de Tampo et al. (2014). En effet les valeurs de la conductivité variaient entre 554 et $4460 \mu \mathrm{S} / \mathrm{cm}$. Toutefois, les valeurs obtenues au cours de la présente étude sont comparables à celles mesurées par Adejuwon et Mbuk (2011). Elles variaient entre 22 et $315 \mu \mathrm{S} / \mathrm{cm}$ au niveau des puits. Le $\mathrm{pH}$ de l'eau est acide avec des valeurs comprises entre 5,17 et 6,88 pour une moyenne de $5,83 \pm 0,6$. Cette acidité des puits est très proche de celle mesurée par Bawa et al. (2008). Les nitrates représentent les anions les plus dominants et la concentration varie de 4,3 à $127 \mathrm{mg} / \mathrm{L}$ avec une moyenne de 45,30 $\pm 40,80 \mathrm{mg} / \mathrm{L}$. L'azote est fixé à l'origine à partir de l'atmosphère et ensuite minéralisé par les bactéries du sol en ammonium. Les sources anthropiques d'azote comprennent l'azote du sol, les engrais, les eaux usées, les fosses septiques, les déchets animaux, les engrais verts et les résidus de plantes. Dans des conditions aérobies, l'azote est finalement convertit en nitrate par les bactéries nitrifiantes (Tindall et al., 1995). Six puits présentent des valeurs en nitrates hors normes (Tableau 2). Parmi ces puits, la moitié se retrouve dans les arrondissements ruraux et la seconde moitié dans le seul arrondissement urbanisé. Cette augmentation de la concentration en nitrates serait liée à l'infiltration des eaux usées ou l'usage des engrais chimiques à proximité de ces puits (Mpakam, 2009). Sa forte concentration dans l'eau potable est toxique et provoque le syndrome du bébé bleu ou méthémoglobinémie chez les enfants et des carcinomes gastriques (Gilly et al., 1984). Les composés suivants présentent des moyennes suivantes : les nitrites $(0,072 \pm 0,14 \mathrm{mg} / \mathrm{L})$, les sulfates $(6,616 \pm 7,26 \mathrm{mg} / \mathrm{L})$, les phosphates $(0,751 \pm 0,61$ $\mathrm{mg} / \mathrm{L})$, les fluorures $(0,142 \pm 0,13 \mathrm{mg} / \mathrm{L})$, les chlorures $(17,75$ et $67,45 \mathrm{mg} / \mathrm{L})$ et les carbonates $(37,870 \pm$ $17,22 \mathrm{mg} / \mathrm{L})$. Ces composés présentent des valeurs en dessous des normes (Tableau 3). Les moyennes des cations étudiés sont les suivants : le calcium $(28,27 \pm$ $18,06 \mathrm{mg} / \mathrm{L})$, le magnésium $(13,63 \pm 4,62 \mathrm{mg} / \mathrm{L})$ et l'ammonium $(0,193 \pm 0,28 \mathrm{mg} / \mathrm{L})$. Deux puits (Pb8 et $\mathrm{Pb} 11)$ présentent des concentrations en ammonium au delà des normes (Tableau 2 et 3). L'eau de ces puits est donc impropre à la consommation humaine. Le Titre Hydrotimétrique ou dureté de l'eau est provoquée par le calcium dissous et dans une moindre mesure par le magnésium. Elle présente des valeurs comprises entre 54 et $232 \mathrm{mg} / \mathrm{L}$ avec une moyenne de 107,8 $\mathrm{mg} / \mathrm{L}$. Selon Sawyer et Mc Carty (1967), l'eau est douce au niveau des puits $\mathrm{Pb} 12$ et $\mathrm{Pb} 14$, dure dans les puits $\mathrm{Pb} 8$ et $\mathrm{Pb} 9$ et légèrement dure dans le reste des puits (Tableau 2 et 4). Ces valeurs sont toutes inférieures à la valeur maximale admissible recommandée par l'Organisation Mondiale de la Santé (2011). Toutefois, un seul puits (Pb9) présente une eau dont la valeur de la dureté est supérieure à celle admissible par les normes béninoises. Elle ne présente pas d'effet majeur pour la santé humaine, cependant elle peut entrainer une consommation excessive de savon et un dépôt de tartre dans les ustensiles de cuisine.

Tableau 3 : Comparaison des moyennes des paramètres physico-chimiques avec les normes

\begin{tabular}{|c|c|c|c|c|c|c|}
\hline Paramètres & Unité & Normes OMS & Normes béninoises & Minimum & Maximum & Moyenne + Ecart-type \\
\hline $\mathrm{Ph}$ & - & $6,5-8,5$ & $6,5-8,5$ & 5,17 & 6,88 & $5,83 \pm 0,6$ \\
\hline$T$ & ${ }^{\circ} \mathrm{C}$ & - & - & 27,6 & 28,7 & $28,15 \pm 0,32$ \\
\hline CE & $\mu S / \mathrm{cm}$ & - & - & 74,8 & 538,2 & $236,62 \pm 135,54$ \\
\hline $\mathrm{Ca}^{2+}$ & $\mathrm{mg} / \mathrm{L}$ & - & 100 & 9,89 & 74,29 & $28,27 \pm 18,06$ \\
\hline $\mathrm{Mg}^{2+}$ & $\mathrm{mg} / \mathrm{L}$ & - & 50 & 5,81 & 22,92 & $13,63 \pm 4,62$ \\
\hline $\mathrm{NO}_{3}^{-}$ & $\mathrm{mg} / \mathrm{L}$ & 50 & 45 & 4,3 & 127,0 & $45,30 \pm 40,80$ \\
\hline $\mathrm{NO}_{2}^{-}$ & $\mathrm{mg} / \mathrm{L}$ & 3 & 3,2 & 0,011 & 0,598 & $0,072 \pm 0,14$ \\
\hline $\mathrm{NH}_{4}{ }^{+}$ & $\mathrm{mg} / \mathrm{L}$ & - & 0,5 & 0,009 & 1,087 & $0,193 \pm 0,28$ \\
\hline $\mathrm{PO}_{4}^{3-}$ & $\mathrm{mg} / \mathrm{L}$ & - & - & 0,22 & 2,84 & $0,751 \pm 0,61$ \\
\hline $\mathrm{SO}_{4}^{2-}$ & $\mathrm{mg} / \mathrm{L}$ & 250 & 500 & 0,50 & 26,00 & $6,616 \pm 7,26$ \\
\hline $\mathrm{Cl}^{-}$ & $\mathrm{mg} / \mathrm{L}$ & 250 & 250 & 17,750 & 67,450 & $31,772 \pm 11,94$ \\
\hline $\mathrm{HCO}_{3}^{-}$ & $\mathrm{mg} / \mathrm{L}$ & - & - & 13,72 & 83,87 & $37,870 \pm 17,22$ \\
\hline TH & $\mathrm{mg} / \mathrm{L}$ & $100-500$ & 200 & 54 & 232 & $107,8 \pm 40,63$ \\
\hline $\mathrm{F}^{-}$ & $\mathrm{mg} / \mathrm{L}$ & 1,5 & 1,5 & 0,01 & 0,52 & $0,142 \pm 0,13$ \\
\hline
\end{tabular}




\section{Lagnika et al. J. Appl. Biosci. 2014. Caractéristiques physico-chimiques de l'eau des puits dans la}

commune de Pobè, Benin

Tableau 4 : Qualité de l'eau des puits selon sa dureté (Sawyer et Mc Carty, 1967)

\begin{tabular}{llll}
\hline Classe de qualité de l'eau & Dureté totale en $\mathbf{~ m g} / \mathbf{L}$ & Nombre de puits & Pourcentage $(\%)$ \\
\hline Douce & $<70$ & 2 & 13,33 \\
\hline Légèrement dure & $75-150$ & 11 & 73,33 \\
\hline Dure & $150-300$ & 2 & 13,33 \\
\hline Très dure & $>300$ & 0 & 0 \\
\hline
\end{tabular}

Corrélations entre les différents paramètres physico-chimiques de l'eau : L'Analyse en Composantes Principales réalisée sur les 14 paramètres physico-chimiques montre que les deux premiers axes cumulent $66,8 \%$ de la variance totale (le premier axe explique $43,39 \%$ et le deuxième axe 23,41 $\%$ de cette variance). L'axe 1 est fortement corrélé du côté positif avec la conductivité électrique, le $\mathrm{pH}$, les nitrates, les nitrites, les sulfates, la dureté totale, les chlorures, les bicarbonates et le calcium. Cet axe exprime à la fois la minéralisation et la pollution organique de l'eau. La mesure de la conductivité pourrait donc suffire pour prédire la qualité de l'eau en ce qui concerne les paramètres précités. Ceci fournit un moyen plus simple et plus rapide pour le suivi de la qualité de l'eau dans une région. Les résultats obtenus sont similaires à ceux de Sunitha et al. (2005) et Raman et Geetha (2005) qui ont montré que la qualité de l'eau souterraine peut être prédite avec précision seulement par la mesure de la conductivité électrique. A l'inverse, l'axe 2 est fortement corrélé du côté négatif avec la température et les fluorures et corrélé du côté positif avec les chlorures et les phosphates. Cet axe exprime moins la minéralisation de l'eau comparativement à l'axe 1. L'ammonium et le magnésium sont moins corrélés avec les deux premiers axes (Fig. 2).

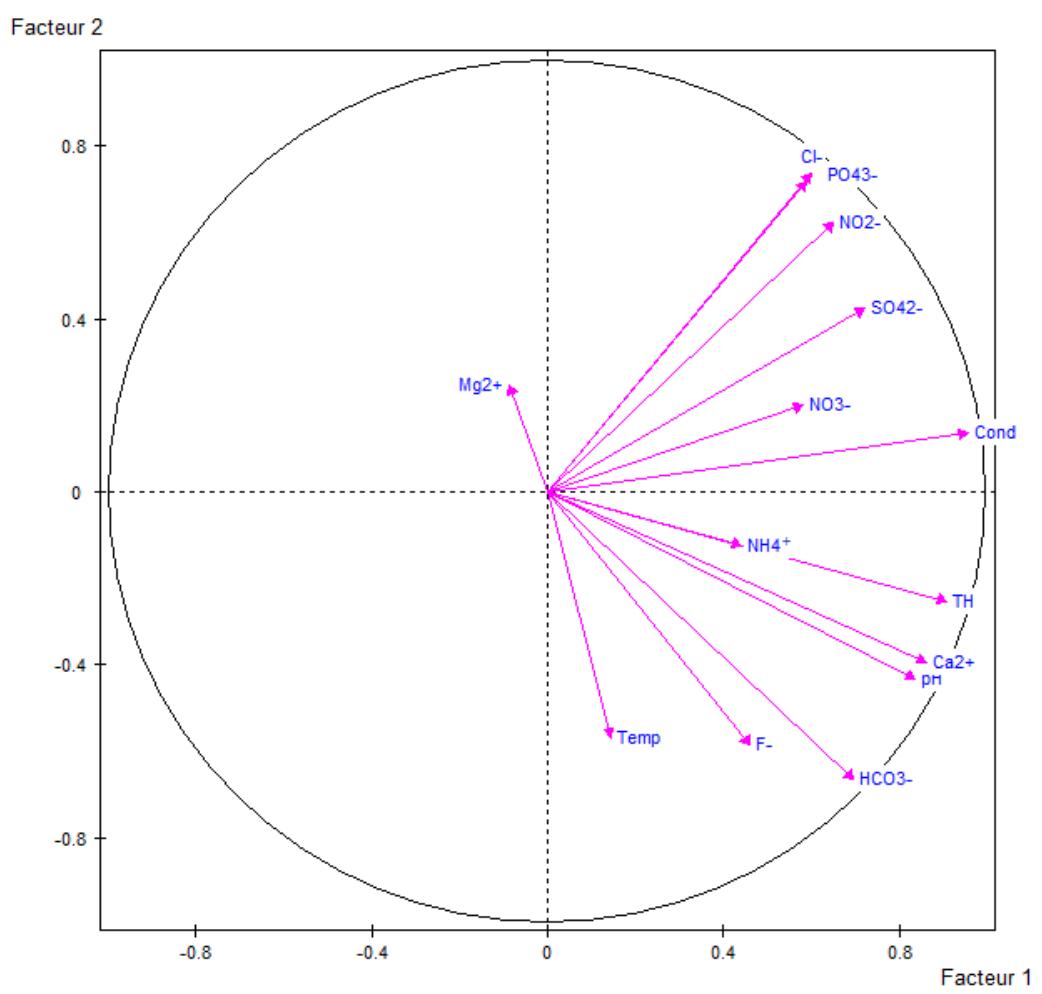

Figure 2 : Projection des paramètres physico-chimiques de l'eau des puits sur les deux premiers axes de l'ACP. Cond : conductivité ; Temp : température 


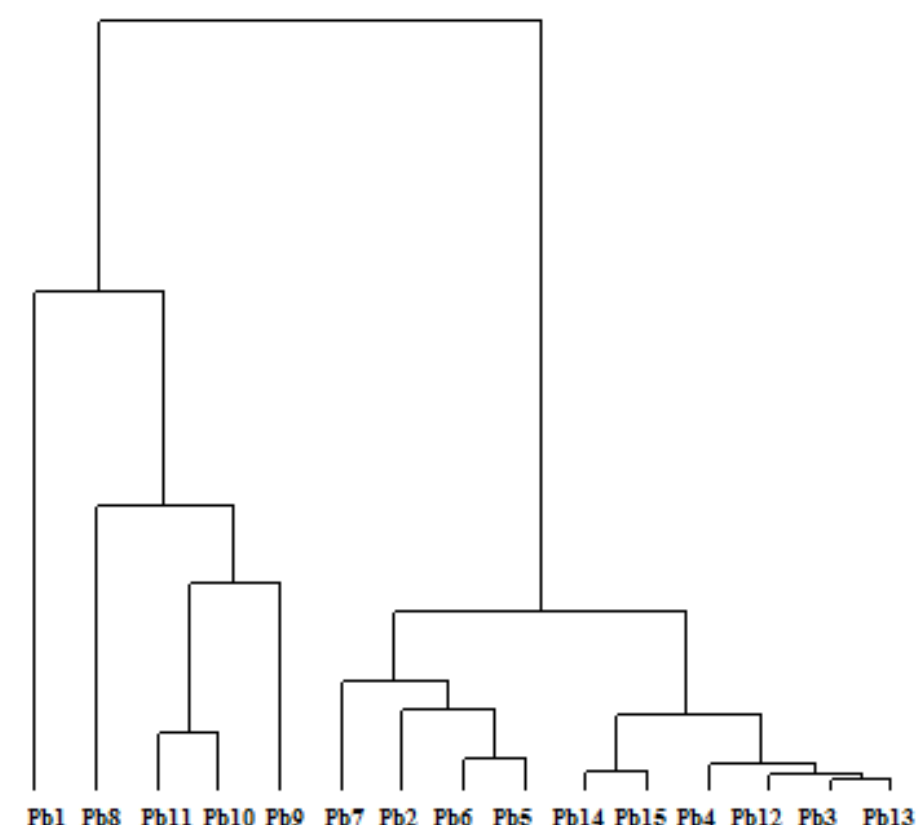

Figure 3 : Dendrogramme représentant la classification hiérarchique ascendante des 15 puits obtenue à partir des paramètres physico-chimiques de l'eau

Typologie des stations: Le dendrogramme représentant la classification hiérarchique des puits (Fig. 3) obtenue à partir des données physicochimiques fait apparaître à un premier niveau de partition trois groupes de stations :

-Groupe 1: il est constitué essentiellement du puits $\mathrm{Pb} 1$. La conductivité électrique est de $538,2 \mu \mathrm{S} / \mathrm{cm}$ et la concentration en nitrates de $91,7 \mathrm{mg} / \mathrm{L}$. L'eau est minéralisée accompagnée d'une pollution azotée.

-Groupe 2: il regroupe les puits $\mathrm{Pb} 8, \mathrm{~Pb} 9, \mathrm{~Pb} 10$ et $\mathrm{Pb} 11$. La conductivité électrique est en moyenne de $335,6 \mu \mathrm{S} / \mathrm{cm}$ et la concentration moyenne en nitrates est de $52,3 \mathrm{mg} / \mathrm{L}$. L'eau est peu minéralisée avec une pollution azotée qui se manifeste par une concentration élevée en nitrates au niveau des puits $\mathrm{Pb} 9, \mathrm{~Pb} 10$ et une concentration en ammonium hors normes au niveau des puits $\mathrm{Pb} 8$ et $\mathrm{Pb} 11$ (Tableau 2).

\section{CONCLUSION}

Au terme de cette étude, les caractéristiques physicochimiques de l'eau des puits ont été déterminées dans la commune de Pobè. Parmi les quatorze paramètres mesurés, les concentrations en nitrates et en ammonium sont au dessus des normes relatives à la qualité de l'eau de boisson dans la majorité des puits.
-Groupe 3: il réunit la majorité des puits (10) et comporte deux sous-groupes. La conductivité moyenne est de $166,85 \mu \mathrm{S} / \mathrm{cm}$ et la concentration moyenne en nitrates est de $37,86 \mathrm{mg} / \mathrm{L}$. L'eau est en général très peu minéralisée et sans pollution azotée.

Le sous-groupe 3-a rassemble les puits $\mathrm{Pb} 2, \mathrm{~Pb} 5, \mathrm{~Pb} 6$ et $\mathrm{Pb7}$. La conductivité moyenne est de $266,175 \mu \mathrm{S} / \mathrm{cm}$ et la concentration en nitrates de $71,275 \mathrm{mg} / \mathrm{L}$ en moyenne. L'eau est peu minéralisée et caractérisée par une pollution azotée excepté le puits $\mathrm{Pb} 7$.

Le sous-groupe 3-b rassemble les puits $\mathrm{Pb} 3, \mathrm{~Pb} 4$, $\mathrm{Pb} 12, \mathrm{~Pb} 13, \mathrm{~Pb} 14$ et $\mathrm{Pb} 15$. La conductivité électrique moyenne est de $100,6 \mu \mathrm{S} / \mathrm{cm}$ et la concentration en nitrates de $15,58 \mathrm{mg} / \mathrm{L}$. L'eau est très peu minéralisée avec de très faibles concentrations en ions azotés.

La pollution serait liée aux activités anthropiques notamment l'infiltration des eaux usées et l'utilisation des engrais chimiques en agriculture. Elle constitue un risque sanitaire pour les populations qui sont tributaires de l'eau de puits pour leur besoin. Pour limiter la pollution de l'eau des puits, les mesures suivantes sont 
à préconiser : isoler les nappes profondes des nappes superficielles par un cuvelage résistant, protéger le toit des apports extérieurs, installer les ouvrages d'assainissement à l'aval hydraulique des puits, éloigner les dépôts de déchets des puits, réduire

\section{REMERCIEMENTS}

Les auteurs remercient la Commission de l'Union Économique et Monétaire Ouest Africaine, le Ministère de l'Enseignement Supérieur et de la Recherche Scientifique du Bénin ainsi que les autorités du Service

\section{RÉFÉRENCES BIBLIOGRAPHIQUES}

Adejuwon J. O., Mbuk C. J., 2011. Biological and physiochemical properties of shallow wells in Ikorodu town, Lagos Nigeria. Journal of Geology and Mining Research. Vol. 3(6), pp. 161-168.

Bani G., 2006. Monographie de la Commune de Pobè, Afrique Conseil, $40 \mathrm{p}$.

Bawa L. M., Tchakala I., Djanéyé-Boundjou G., 2008. Détermination de la demande en chlore des eaux de puits et de forages d'un quartier périurbain de la ville de Lomé : incidence sur la désinfection. Journal des Sciences et Technologie. Vol. $7 n^{\circ} 2$ pp.19-24.

Belkhiri L., Boudoukha A., Mouni L., Baouz T., 2010. Multivariate statistical characterization of groundwater quality in Ain Azel plain, Algeria. African Journal of Environmental Science and Technology. Vol. 4(8), pp. 526-534.

Comlanvi F.M., 1994. Amélioration de la qualité des eaux de puits dans la ville de Cotonou : cas de quelques quartiers. Mémoire de fin de formation DIT, aménagement, protection de l'environnement, CPU, UNB, Bénin, 78p.

Dégbey C., Makoutode M., Ouendo E-M., Fayomi B., De Brouwer C., 2008. La qualité de l'eau de puits dans la commune d'Abomey-Calavi au Bénin. Environnement, Risques et Santé. Vol. 7, $n^{\circ} 4$, pp 279-283.

Eblin S. G., Sombo A. P., Soro G., Aka N., Kambiré O., Soro N., 2014. Hydrochimie des eaux de surface de la région d'Adiaké. J. Appl. Biosci. 75: 6259-6271.

El-Naqa A., Al-Momani M., Kilani S., Hamouri N., 2007. Groundwater deterioration of shallow groundwater aquifers due to overexploitation in northeast Jordan. Clean Soil, Air, Water 35:156-166. l'utilisation des engrais chimiques. Cette étude a également montré l'importance de l'utilisation des analyses multivariées pour caractériser les puits dans le cadre des programmes de planification et de suivi de la qualité de l'eau dans une région.

de l'Eau du Borgou pour leur soutien technique et financier. Ils expriment leur gratitude au Professeur Claude BOUTIN qui a initié et accompagné cette recherche.

Fatombi K. J., Ahoyo T. A., Nonfodji O., Aminou T., 2012. Physico-Chemical and Bacterial Characteristics of Groundwater and Surface Water Quality in the Lagbe Town: Treatment Essays with Moringa oleifera Seeds. Journal of Water Resource and Protection. 4: 1001-1008.

Gilly G., Corrao G., Favilli S., 1984. Concentrations of nitrates in drinking water and incidence of gastric carcinomas: First descriptive study of the Piemonate region Italy. Sci. Total Environ, 34: $35-37$.

Hawa S., 2002. Analyse physico-chimique et bactériologique au L.N.S. des eaux de consommation de la ville de Bamako durant la période 2000 et 2001. Thèse de Doctorat d'état en Pharmacie, $77 \mathrm{p}$.

Ingabiré Zannou F., 2006. Phénomène des postes d'eau autonomes privés au Bénin : Diagnostic, impacts et solutions alternatives. Mémoire de DESS-MEQuE, FAST-UAC. $86 p+$ annexes.

INSAE, 2003. Institut National de Statistique et de l'Analyse Economique, recensement général de la population et de l'habitat 3. $42 \mathrm{p}$.

Makoutodé M., Assani A. K., Ouendo E. M., Agueh V. D., Diallo P., 1999. Qualité et mode de gestion de l'eau de puits en milieu rural au Bénin : cas de la sous préfecture de Grand-Popo. Médecine d'Afrique noire, 46 (11): 528-534.

Mpakam H. G., 2009. Vulnérabilité à la pollution des ressources en eaux à Bafoussam et incidences socio-économiques et sanitaires : modalités d'assainissement. Thèse de Doctorat, Université de Yaoundé I, $267 \mathrm{p}$.

Nwala C. O., Akaninwor J. O., Abbey B. W., 2007. Physico-chemical parameters of monopumps and well waters in Igbo Etche. J. Nig. Environ. Soc., 4(1): 78-87. 
OMS, 2011. Directives de qualité pour l'eau de boisson. Quatrième édition. Publication Organisation Mondiale de la Santé. Genève, Suisse. pp.307-447.

Raman S. B. K., Geetha G., 2005. Correlation analysis and prediction of characteristic parameters and water quality index of ground water. Pollut. Res., 24:197-200.

Rodier J., 2009. L'analyse de l'eau. Dunod, gème édition, $1526 \mathrm{p}$.

Sawyer C. N., Mc Carty P. L., 1967. Chemistry of Sanitary Engineers. 2nd Edn., Mc Grow Hill, New York, 518 pp.

Scanlon B. R., Reedy R. C., Stonestrom D. A., Prudic D. D. D. E., Dennehy K. F., 2005. Impact of land use and land cover change on groundwater recharge and quality in the southwestern US. Global Biol., 11: 1577-1593.
Servais P., Billen G., Garcia-Armisen T., George I., Goncalvez A., Thibert S., 2009. La contamination microbienne dans le bassin de la Seine. Edition. Agence de l'Eau Seine Normandie. 50 pp. ISBN: 978-2-918251-07-1.

Sunitha V., Sudarshan V., Reddy B. R., 2005. Hydrogeochemistry of groundwater, Gooty area, Anantapur district Andhra Pradesh, India. Poll. Res., 24: 217-224.

Tampo L., Ayah M., Kodom T., Tchakala I., Boguido P., Bawa L., Djaneye B., 2014. Impact de la demande en chlore et de la chloration sur la désinfection des eaux de puits des quartiers de Lomé. J. Appl. Biosci.75: 6272-6281.

Tindall J. A., Petrusak R. L., Mc Mohan P. B., 1995. Nitrate transport and transformation process in unsaturated porous media. J. Hydrol., 169, 5194. 